\title{
LOS CICLOS INICIALES OPTATIVOS DEL CENTRO UNIVERSITARIO DE LA REGIÓN ESTE: INNOVACIÓN Y FLEXIBILIDAD CURRICULAR EN LA UNIVERSIDAD DE LA REPÚBLICA, URUGUAY
}

\author{
Pilar Rodríguez, Laura Brum, Rossana Cantieri, Paula \\ Laporta y Natalia Verrastro ${ }^{1}$
}

\section{RESUMEN}

La Universidad de la República (UR) de Uruguay ha comenzado un proceso de descentralización y cambios muy profundos. En este marco se crean los Ciclos Iniciales Optativos (CIO), una vía alternativa de ingreso a la universidad, que tienen como objetivo facilitar el acceso a la educación terciaria y evitar la desvinculación.

Este artículo describe la implementación y valoración de los $\mathrm{CIO}$ en el marco de la descentralización y creación del Centro Universitario de la Región Este.

Las principales características de los CIO como programa innovador son la flexibilidad curricular y la integración de saberes mediante el abordaje interdisciplinario.

Finalmente, se da cuenta de las fortalezas y desafíos del programa, así como de las mejoras a implementar.

Palabras clave: ciclos iniciales optativos, trayectorias flexibles, innovación educativa, descentralización universitaria.

\section{THE OPTIONAL INITIAL CYCLES IN THE UNIVERSITY CENTRE OF THE EAST REGION: INNOVATION AND CURRICULAR FLEXIBILITY IN THE REPUBLIC UNIVERSITY, URUGUAY}

\section{ABSTRACT}

The Republic University of Uruguay (UR) has begun a process of decentralization and deep changes. In this framework the Optional Initial Cycles (CIO, for the Spanish acronym) are created as an alternative route of entry into the university, which aim to facilitate access and decrease student desertion.

This article describes the implementation and evaluation of the $\mathrm{CIO}$ in the context of university decentralization and the creation of the Eastern University Regional Centre.

CIO's main characteristics as an innovative program are the curriculum flexibility and the integration of knowledge through an interdisciplinary approach.

Finally, it describes the strengths and challenges of the program and the implementation of improvements.

Keywords: optional initial cycles, flexible paths, educational innovation, university decentralization.

1 Centro Universitario de la Región Este. Universidad de la República. Maldonado, Uruguay. Contacto: prodriguez@curemaldonado.edu.uy 


\section{LOS CICLOS INICIALES OPTATIVOS DEL CENTRO UNIVERSITARIO DE LA REGIÓN ESTE: INNOVACIÓN Y FLEXIBILIDAD CURRICULAR EN LA UNIVERSIDAD DE LA REPÚBLICA, URUGUAY}

\section{La Universidad de la República y la nueva reforma universitaria}

La Universidad de la República² se funda a mediados del siglo XIX. Hacia el año 1952 se establece el cogobierno universitario mediante la Constitución de la República. Con esta reforma constitucional se instituía la participación en la toma de decisiones de los tres órdenes: docentes, estudiantes y egresados. La Ley Orgánica de 1958 reúne y unifica todo el ciclo reformador.

En los últimos años la UR se ha embarcado en un nuevo proceso de reformas. Un eje de esas reformas apunta a lo que se denomina descentralización, es decir, llevar la educación universitaria a todo el país, dejando atrás la centralización de la formación universitaria en la capital del país.

En el año 2007 el Consejo Directivo Central (CDC), órgano máximo de la UR, tomó varias resoluciones que pusieron en marcha el proceso de reforma. En abril de ese año el CDC afirma que "la flexibilización, diversificación y articulación de la enseñanza tienen como objetivo facilitar el acceso a la educación terciaria y universitaria, profundizar el proceso de democratización de la enseñanza superior y su universalización, y mantener los vínculos de los estudiantes con el sistema" (Universidad de la República, 2007).

2 La Universidad de la República es el ente público encargado de administrar educación universitaria en el Uruguay y después de 160 años de fundada es la única universidad pública en el territorio nacional. 


\section{La descentralización y la creación del Centro Universitario de la Región Este}

La UR nació como una universidad centralizada en la capital y se mantuvo de esa manera por más de un siglo. Sin embargo, la creciente demanda de formación universitaria hizo que la UR se planteara en varias ocasiones su descentralización para poder cumplir con el objetivo de democratizar el acceso a la universidad, aunque las iniciativas en tal sentido habían sido muy puntuales.

En la sesión extraordinaria del 17 de julio de 2007, el CDC toma varias decisiones importantísimas para el desarrollo de la universidad en el interior de la república: la creación de la Comisión Coordinadora del Trabajo de la Universidad en el Interior, el impulso a la conformación de los Programas Regionales de Enseñanza Terciaria (PRET) y la creación del Centro Universitario de la Región Este.

Los PRET comienzan a consolidarse, no solo como polos regionales que democratizan el acceso a la educación universitaria, sino como centros comprometidos con el propósito de potenciar el desarrollo regional (Comisión Coordinadora del Interior, 2008).

El CDC resuelve establecer ejes prioritarios de desarrollo para cada región. En el caso de la región Este -que comprende los departamentos de Maldonado, Rocha, Treinta y Tres y Lavalleja (en proceso de integración) se indican como prioritarias las temáticas vinculadas al medio ambiente, biodiversidad, ecología, costa, pesca y turismo (Universidad de la República, 2009).

\section{Los Ciclos Iniciales Optativos}

La propuesta de los Ciclos Iniciales Optativos (CIO) se enmarca en este proceso de transformación iniciado por la UR con el objetivo de lograr la generalización y diversificación de la enseñanza terciaria pública.

Estos ciclos iniciales, que constituyen una innovación educativa dentro de la universidad, tienen como finalidad contribuir a la 
flexibilización y articulación de los procesos de formación curricular. Además, representan una estrategia para el apoyo, orientación y fortalecimiento de las capacidades de los estudiantes, evitando así, uno de los principales obstáculos en este proceso educativo: la desvinculación (Comisión Sectorial de Enseñanza, 2010).

Se impulsa la implementación de los CIO particularmente en las sedes del interior, ya que constituyen una vía alternativa de ingreso a la universidad (primer año) y operan como programas que contribuyen a democratizar territorialmente y a generalizar el acceso a la educación superior. Asimismo, pretenden orientar a los estudiantes en la consolidación de su opción vocacional, el conocimiento de la UR, el fortalecimiento de conocimientos y desarrollo de capacidades de autocrítica y autoevaluación, promoviendo metodologías de enseñanza activas, dialogantes y participativas.

Se aprueban tres Ciclos Iniciales Optativos, dos de orientación Ciencia y Tecnología (uno implementado en la sede del CURE y otro en la Regional Norte) y uno de orientación Social (implementado en el CURE). De esta manera, los CIO se insertan en la estructura académica de los Centros Universitarios Regionales (CENURES), complementando las estructuras de facultades vigentes en la UR, ya que no pretenden competir con estas, sino agregar ofertas curriculares y académicas. Además, estos CiO vienen trabajando desde una mirada diferente, adoptando los cambios anteriormente mencionados, y constituyéndose en verdaderos planes piloto, parte esencial en la reforma universitaria.

El primer año en la vida del estudiante universitario es un momento de transición donde algunos no están totalmente definidos en cuanto a su vocación, no conocen en profundidad las propuestas de formación que les brinda la UR, razón por la cual es importante un primer ciclo flexible, donde puedan experimentar y aproximarse a su vocación, adquirir cierta preparación y acumular créditos para su posterior formación. De esta manera, durante el primer año de ingreso a la UR los estudiantes tienen oportunidad de ir ajustando su trayectoria curricular de acuerdo con sus intereses y posibilidades, acumulando los créditos obtenidos durante los cursos, aun si deciden 
cambiar de opción de formación. En este proceso de elección de sus trayectorias, los alumnos encuentran el apoyo fundamental de los docentes tutores, que van construyendo con ellos esta malla curricular.

La experiencia de los CIO en el CURE busca, además, una formación integral del estudiante universitario, promovida desde la UR como parte de la formación y el compromiso ético de todo profesional universitario. Esta integralidad implica tres grandes conceptos: interdisciplinariedad, articulación de las funciones universitarias (investigación, docencia y extensión) e integración de los actores involucrados, en referencia directa a los vínculos con la comunidad. La UR se ha comprometido con incluir en el currículo estas actividades, constituyéndose en parte fundante del proceso de transformación universitaria. Se persigue la articulación del conocimiento y la interacción con la sociedad, desde una perspectiva crítica y comprometida, buscando mejorar la calidad de vida de las comunidades y el intercambio de saberes entre ambas (Comisión Sectorial de Extensión y Actividades en el Medio, 2007).

Por último, debe destacarse su carácter experimental, y consecuentemente, se hace necesario realizar tanto la evaluación continua como el seguimiento permanente de la propuesta. Por este motivo, en el contexto del equipo de trabajo de la Unidad de Apoyo a la Enseñanza (UAE) y del Equipo de Apoyo Docente de los Ciclos Iniciales Optativos (EAD-CIO) del CURE se llevó a cabo el diseño, implementación y valoración del desempeño docente y de los cursos impartidos del CIO, por parte de los estudiantes del CURE. Algunos de los resultados de estos estudios son presentados en este artículo.

\section{La propuesta educativa que plantean los Ciclos Iniciales Optativos}

El CIO del CURE se estructura en dos grandes orientaciones: la social y la científico-tecnológica. Surgen en forma independiente, pero en la práctica funcionan como un ciclo único. 
El CIO orientación científico-tecnológica tiene como objetivo general promover la formación terciaria y superior con un abordaje interdisciplinario e integrador de funciones, con una visión global, pero también de acuerdo con las necesidades y el desarrollo de la región.

El CIO orientación social establece como objetivos ofrecer a los estudiantes una formación panorámica en las ciencias sociales y humanas, brindándoles un conjunto de elementos teóricos, metodológicos, así como técnicas y herramientas básicas para el análisis social.

Ambos ciclos proponen un sistema de créditos organizado por áreas, módulos y asignaturas. Además se incorpora el concepto de trayectoria sugerida que hace alusión a un posible itinerario que tenga en cuenta la funcionalidad de la selección del estudiante en relación con la carrera en la cual se insertará.

Los CIO se desarrollan en un sistema de 90 créditos, en un espacio temporal de un año de estudios universitarios, sobre un curriculum flexible, organizado en áreas de formación. Cada área está estructurada en módulos, los que a su vez contienen asignaturas. Las áreas de formación propuestas específicamente son: básica, técnicometodológica e interdisciplinaria.

Los estudiantes que cursan los $\mathrm{CIO}$ deben tomar asignaturas de las tres áreas. Además, existen dos asignaturas obligatorias para todos los estudiantes: Introducción a las trayectorias flexibles en la UR (área de formación técnico-metodológica) y Taller interdisciplinario de tópicos regionales (área de formación interdisciplinaria).

La labor de conformar un curriculum flexible no es tarea fácil para un estudiante que proviene de la educación media donde no se le ha brindado la posibilidad de elegir asignaturas. Por tanto, se debe apoyarlos con estrategias de orientación y supervisión.

En primer lugar, es importante que el estudiante se involucre en el armado de su curriculum y que cambie su actitud pasiva, frente a las opciones de formación que se le presentan, a una actitud participativa 
y exploradora, de construcción de su vocación. En segundo lugar, se debe crear una estructura de apoyo para estos estudiantes, y también para los docentes, que en primera instancia puedan estar algo confusos sobre esta propuesta específica, por lo novedosa y flexible. En este caso fue importante la creación del Equipo de Apoyo Docente que, entre otras tareas, cumple el rol de orientar a los estudiantes y docentes en las distintas trayectorias e informar sobre el reconocimiento de estos créditos en las diferentes carreras, facultades y centros universitarios. Por último, la implementación de un curriculum flexible implica que los estudiantes deban contar con un asesoramiento personalizado, por lo tanto, los docentes deben cumplir con su papel de tutores para orientarlos en la construcción de su trayectoria.

\section{Taller interdisciplinario en el marco de los Ciclos Iniciales Optativos}

La propuesta del CIO establece que se deben completar para su aprobación 24 créditos del área interdisciplinaria. La asignatura principal para cumplir este requerimiento es el Taller interdisciplinario de tópicos regionales (TI), de carácter anual y donde se propone el abordaje de temáticas regionales desde un punto de vista interdisciplinario.

El objetivo del TI es abordar temas y problemas de la región, de diversa índole, profundizados desde diferentes miradas disciplinares, con una visión holística e integradora y en el contexto o realidad en la que se insertan (Conde y Sztern, 2010).

El TI implica la utilización de una metodología participativa y de trabajo grupal, lo que constituye un aporte más hacia instancias ricas en intercambio, proyección, planificación, aprendizaje y evaluación permanentes, entre estudiantes y docentes.

Durante el primer semestre los estudiantes son introducidos en los siguientes temas: los principales desafíos ambientales del Uruguay y de la región, la interdisciplinariedad y transdisciplinariedad, los paradigmas del enfoque sistémico, la enseñanza problemática, la investigación participativa y la formulación de proyectos. Esta 
formación les permite identificar los estudios de casos para desarrollar en el segundo semestre donde tendrán que formular un proyecto de investigación, llevarlo a cabo y presentar, finalmente, un informe escrito del trabajo de investigación realizado.

La modalidad de funcionamiento para los casos de estudio implica trabajar en pequeños grupos conformados por estudiantes con diferentes intereses y orientaciones, docentes con distintas formaciones coordinando cada grupo y que uno de ellos actúe como tutor responsable, para luego acordar entre docentes y estudiantes el caso específico de estudio.

Los criterios para el desarrollo de los casos estarán sujetos a una previa coordinación con el equipo responsable de la asignatura. Los estudios pueden ser teóricos, pero lo más adecuado es que desarrollen trabajo de campo y/o laboratorio.

\section{Evaluación de la primera edición de los $\mathrm{CIO}$}

En el año 2010 se implementó por primera vez el programa CiO en el CURE. Se inscribieron un total de 151 estudiantes, de los cuales solo 59 se inscribieron a asignaturas. El escaso tiempo de difusión del programa, unido a un comienzo retrasado de los cursos con respecto al resto de las carreras del centro universitario, hizo que muchos de los inscritos no comprendieran bien la propuesta y, por tanto, desistieran de su intención de cursar.

Al finalizar el primer año de aplicación del programa CIO se realizaron varias evaluaciones sobre su implementación. Una de ellas consistió en consultar la opinión de los estudiantes en relación con sus docentes, las asignaturas y al programa en sí mismo.

Mencionaremos algunos de los puntos más representativos de las percepciones de los estudiantes. Los resultados completos se encuentran en el informe de Rodríguez, Brum, Verrastro et al. (2010).

Uno de los aspectos esenciales fue la comprensión de la propuesta por parte de los estudiantes que cursaron durante ese año, es decir, las posibilidades que les ofrece el programa. Cuando los 
estudiantes fueron consultados sobre este asunto, el 75\% afirmó haber comprendido la propuesta de formación del CIO y las posibilidades que les brindan las distintas trayectorias.

Los estudiantes destacaron positivamente el nivel de exigencia de las asignaturas y la rigurosidad científica con las que fueron dictadas.

En relación con los docentes, un 90\% de los estudiantes destacó la preparación de acuerdo con los requerimientos del curso, un 95\% consideró que preparan adecuadamente sus clases y un $89 \%$ opinó que utilizan diferentes estrategias para el aprendizaje de sus alumnos. Los docentes fueron el recurso mejor valorado dentro del programa. Sin embargo, debemos señalar que el desempeño de los docentes como tutores no obtuvo tan buenos resultados. Un 39\% de los estudiantes consideraron que no fueron bien asesorados por sus tutores.

Ante la pregunta abierta sobre qué es lo más destacable del $\mathrm{CIO}$, los estudiantes mencionaron la flexibilidad y las actividades interdisciplinarias (Rodríguez, Brum, Verrastro et al., 2010).

\section{Fortalezas}

Sin dudas, los CIO son una propuesta que la UR seguirá impulsando y que, seguramente, se implementarán no solo en los Centros Universitarios Regionales, sino en las distintas facultades de la capital. Su principal virtud se centra en ofrecer al estudiante una vía alternativa de ingreso a la universidad, que puede constituir un primer año orientado hacia un área o macro-área. Brinda una formación integrada e integradora de saberes, por lo tanto, deja de lado la tradicional fragmentación en diferentes disciplinas. También permite a estudiantes continuar estudios en un área no relacionada con la orientación del bachillerato del que provienen.

Dado que los CIO funcionan en dos Centros Regionales tienen otras virtudes: acercan la formación universitaria a estudiantes que, de otra forma, no podrían acceder a la universidad, y por otro lado, prolongan el momento en que el estudiante deba dejar su hogar para continuar estudios. De esta manera, se evita que el estudiante deba realizar dos adaptaciones simultáneamente: a un nuevo y más exigente 
288 LOS CICLOS INICIALES OPTATIVOS DEL CENTRO UNIVERSITARIO DE LA REGIÓN ESTE: INNOVACIÓN Y FLEXIBILIDAD CURRICULAR EN LA UNIVERSIDAD DE LA REPÚBLICA, URUGUAY - Pilar Rodríguez, Laura Brum, Rossana Cantieri, Paula Laporta y Natalia Verrastro

sistema educativo (la enseñanza terciaria) y a nuevas condiciones de vida (traslado a la capital).

\section{Desafíos y mejoras para implementar}

Sobre la base de las evaluaciones realizadas en 2010 (Rodríguez, Brum, Verrastro et al., 2010) se proponen varias acciones de mejoras. Algunas de ellas ya se implementaron para la segunda edición. Se sintetizan aquellas que se pueden desarrollar desde el propio programa:

\section{- Asesoramiento a los estudiantes ingresantes}

Es necesaria la comprensión de la propuesta por los futuros estudiantes. Para ello, se difundió la oferta educativa del CURE, el funcionamiento general de la UR y los requisitos para el ingreso, realizando charlas informativas en los centros de educación media de la región este. Además, se realizó un asesoramiento personalizado durante la inscripción, donde se orientó a los estudiantes acerca de la propuesta formativa ofrecida y sus posibles trayectorias en función de los intereses que inicialmente demostraban.

- Apoyo en la construcción del curriculum e ingreso a la universidad

Como ya se mencionó, enfrentarse a la construcción del curriculum puede ser una tarea compleja. Por eso, para lograr una orientación previa a la elección de asignaturas se instrumentó una asignatura denominada "Introducción a las trayectorias flexibles en la UR" que tuvo como objetivos introducir a los estudiantes en torno a las características de nuestra universidad, orientarlos en la construcción de su curriculum flexible y brindarles herramientas para su desempeño.

Esta asignatura se dictó durante la primera semana de clases y combinó exposiciones teóricas y trabajo en taller con los docentes tutores. A cada uno de ellos se les asignó un número aproximado de diez estudiantes.

Esta asignatura ofreció un marco ideal para el conocimiento de los estudiantes entre sí y con sus docentes, siendo a la vez, un buen 
punto de inicio para el trabajo interdisciplinario al que se apunta en este ciclo. También, pretendió ofrecer un panorama más sistematizado y ordenado, que el brindado el año anterior, para acompañar el ingreso de los 312 estudiantes inscritos en esta segunda edición. Del total de estudiantes, 302 inscribieron asignaturas y el número final cursando, pasado el 30 de abril (último plazo para aprobar la asignatura previa con la que se pueden inscribir), fue de 279 estudiantes.

\section{- Tutorías:}

Teniendo en cuenta que un 39\% de los estudiantes consideró que no fue bien asesorado por sus tutores, para la edición 2011 del CIO se implementaron cursos de formación de tutores y asesoramiento para su correcto desempeño. Durante el comienzo del año 2011 se coordinó con el Programa del Respaldo al Aprendizaje (dependiente de la UR) y la Unidad de Apoyo a la Enseñanza del CURE para brindar una formación mínima para desempeñarse como tutor. Asimismo, se establecieron estándares de dimensiones para considerar en la tutoría forma y asiduidad de la comunicación con los tutelados, así como una forma de evaluación periódica de esta actividad.

\section{- Uso de metodologías innovadoras}

Un punto que los estudiantes plantearon como necesario fue el de favorecer el uso de metodologías innovadoras para el aprendizaje. Este aspecto aparece como el único factor destacable de merecer una atención particular porque en el resto de los asuntos, los docentes fueron muy bien valorados.

Las formas de aprender han cambiado, han ido adquiriendo nuevas modalidades, lo cual requiere que el docente adopte y utilice nuevas metodologías para enseñar. A partir de lo expuesto, consideramos necesaria y beneficiosa la implementación de cursos destinados a los docentes sobre dichas metodologías innovadoras. En esa dirección se coordinó para 2011, a través de la UAE, la segunda edición de un curso destinado a los docentes de la región en el que se instruye sobre el empleo de la plataforma educativa de la UR, Espacio Virtual de Aprendizaje (EVA). 


\section{- Reconocimiento y validación}

Los CIO, como propuesta innovadora dentro de la UR, todavía no han logrado el nivel de reconocimiento deseado o esperado en la estructura universitaria. Si bien algunas facultades los han estudiado y aprobado íntegramente como el primer año de sus carreras, son minoritarias. Los CIO proponen un desafío para la estructura universitaria tradicional, organizada en áreas de conocimiento y con una fuerte tradición de facultades y servicios, planteando transversalidad y flexibilidad.

La validación del programa por parte de los distintos consejos y claustros de facultades se transforma en un punto crucial al momento de pensar en la proyección y continuidad futuras de los CIO.

\section{- Difusión}

Es necesaria la divulgación de la experiencia, su discusión y apropiada valoración. Además, la experiencia de los programas CIO debe darse a conocer entre quienes serán futuros estudiantes universitarios, entre quienes lo son actualmente y entre quienes en algún momento lo fueron (docentes y egresados), a modo de conocerla, discutirla, profundizarla y extenderla.

Las valoraciones de las diferentes aplicaciones del programa servirán de insumos para tomar las decisiones necesarias para mejorarlo y realizar las adecuaciones para su implementación en otras circunstancias.

\section{Consideraciones finales}

En primer lugar se resalta la buena aceptación de la propuesta entre los estudiantes, confirmada por el número de inscritos en la primera edición y la duplicación de la matrícula en la segunda. Los CIO se han transformado en la segunda opción de formación con mayor número de estudiantes del CURE.

Los CIO cumplen la función de ser una formación universitaria de calidad que habilita a continuar estudios en diferentes carreras. 
Es necesario continuar con las evaluaciones anuales de cada edición del programa y el seguimiento de los estudiantes para garantizar la calidad de la propuesta e implementar las mejoras necesarias para su perfeccionamiento, así como se ha hecho en la segunda edición.

Es importante la generación de grupos de trabajo conformados por los diferentes órdenes de la UR (docentes, egresados y estudiantes) para discutir y reflexionar sobre el programa CIO y sus implicancias filosóficas y prácticas dentro de la universidad: interdisciplina, flexibilidad, movilidad, entre otras.

Se debe considerar y dar apoyo a las dificultades de los estudiantes para enfrentarse a la conformación de un curriculum flexible, además de orientar sobre el funcionamiento y posibilidades de estudios que brinda el programa. Para lograr estos objetivos se hace necesario reforzar la figura del tutor.

Finalmente, es relevante difundir la experiencia del CIO de modo que se evalúe la posible implementación en el resto de las facultades y centros universitarios, ya que los CIO tienen el potencial de formar un nuevo estudiante y posterior egresado con una visión más amplia y no compartimentada por disciplinas, tan demandada en la formación universitaria para el siglo XXI.

\section{Referencias bibliográficas}

Comisión Coordinadora del Interior. Programas regionales de enseñanza terciaria 2008-2010 y su proyección al 2020 (2008) Serie Documentos de Trabajo CCI N 1 . Universidad de la República. Montevideo, p. 6.

Comisión Sectorial de Extensión y Actividades en el Medio. Programas integrales: concepción y gestión. Aportes para el debate universitario. (2007) Equipo de Trabajo sobre Programas Integrales. Universidad de la República. Montevideo, pp. 4-7.

Comisión Sectorial de Enseñanza. Ciclos Iniciales Optativos. Una alternativa para el ingreso a la Universidad de la República. (2010) Montevideo. Universidad de la República, p. 3. Disponible en: //www.cse.edu.uy/ node/139. 
292 LOS CICLOS INICIALES OPTATIVOS DEL CENTRO UNIVERSITARIO DE LA REGIÓN ESTE: INNOVACIÓN Y FLEXIBILIDAD CURRICULAR EN LA UNIVERSIDAD DE LA REPÚBLICA, URUGUAY - Pilar Rodríguez, Laura Brum, Rossana Cantieri, Paula Laporta y Natalia Verrastro

Conde, D. y Sztern, J. (2010) Taller interdisciplinario sobre tópicos regionales para CIO-CURE. $2^{\circ}$. Documento de trabajo. Documento interno, p. 2.

Rodríguez, P.; Brum L.; Verrastro, et al. (2010) Informe de la evaluación del Ciclo Inicial Optativo del Centro Universitario de la Región Este. Disponible en: http://www.cure.edu.uy/index.php/cio.

Universidad de la República (2007) Hacia la Reforma Universitaria. Resoluciones del Consejo Directivo Central de la Universidad de la República 31-03-07/1408-07. N. ${ }^{\circ}$. Montevideo. Departamento de Publicaciones, p. 29.

Universidad de la República (2009) Hacia la Reforma Universitaria. La Universidad en el Interior. N. ${ }^{\circ}$. Montevideo. Rectorado Universidad de la República, p.16.

Recibido: 30/09/2011

Aceptado: 12/10/2011 